\title{
Reduction in Morning Blood Pressure Is a Key Factor for Ameliorating Urinary Albumin Excretion in Patients With Morning Hypertension Irrespective of Treatment Regimen
}

\author{
Hisashi Kai, MD, PhD; Masashi Kaneyuki, MD; Miwako Shihara, MD, PhD; \\ Yasuyuki Toyama, MD; Yoshiaki Mitsutake, MD; Hidekazu Umei, MD, PhD; \\ Ken Kusaba, MD, PhD; Tamenobu Ueda, MD; Hisashi Adachi, MD, PhD; \\ Tsutomu Imaizumi, MD, PhD for the MAPPY Study Investigators
}

\begin{abstract}
Background: The Morning Hypertension and Angiotensin Receptor Blocker/Hydrochlorothiazide Combination Therapy (MAPPY) study has shown that losartan/hydrochlorothiazide (HCTZ) combination is superior to high-dose losartan in not only reducing morning systolic blood pressure (SBP) but also ameliorating urinary albumin excretion (UAE) after 3-month treatment. The purpose of the present study was to investigate factors associated with UAE reduction in on-treatment patients with morning hypertension.
\end{abstract}

\begin{abstract}
Methods and Results: A total of 95 patients registered in the MAPPY study were analyzed. Patients were treated with either a losartan/HCTZ combination regimen $(n=47)$ or a high-dose losartan regimen $(n=48)$. Three-month treatment significantly reduced morning SBP, evening SBP, and clinic SBP $(P<0.001, P<0.05$, and $P<0.01$, respectively). UAE and serum uric acid were significantly decreased $(\mathrm{P}<0.01$ for both) without the change in estimated glomerular filtration rate. Multiple linear regression analysis indicated that \%morning SBP reduction and baseline UAE were independent determinants of the UAE reduction ( $P=0.001$ for both). After adjustments for the reduction in morningevening SBP difference, baseline UAE, and \%uric acid reduction, estimated \%UAE reduction level was positively correlated with the tertiles of the increasing \%morning SBP reduction level ( $P=0.031$ for trend). Moreover, subgroup analysis showed that morning SBP reduction was an independent determinant of UAE reduction in both treatment regimens.
\end{abstract}

Conclusions: Reduction in morning SBP was a key factor in UAE reduction in patients with morning hypertension, irrespective of treatment regimen. (Circ J 2013; 77: 1551-1557)

Key Words: Albuminuria; Angiotensin II receptor blocker; Diuretics; Home blood pressure measurement; Morning hypertension

$\mathbf{R}$ ecently, we conducted the Morning Hypertension and Angiotensin Receptor Blocker/Hydrochlorothiazide Combination Therapy (MAPPY) study to compare the efficacy of losartan/hydrochlorothiazide (HCTZ) combination and high-dose losartan in controlling morning hypertension. ${ }^{1}$
In the MAPPY study, losartan/HCTZ combination was superior to high-dose losartan in the primary endpoint: morning systolic blood pressure (SBP) reduction after 3-month treatment. Unexpectedly, urinary albumin excretion (UAE) reduction, a secondary endpoint, was significantly greater in patients

Received August 26, 2012; revised manuscript received January 9, 2013; accepted January 23, 2013; released online March 2, 2013 Time for primary review: 19 days

Department of Internal Medicine, Division of Cardio-Vascular Medicine (H.K., M.K., M.S., Y.T., Y.M., H.U., K.K., T.U., T.I.), Department of Community Medicine (H.A.), Kurume University School of Medicine, Kurume; Division of Cardiology, Tanushimaru Central Hospital, Kurume (Y.M.); Division of Cardiology, Yokokura Hospital, Miyama (H.K.); Division of Cardiovascular Medicine, Omuta City General Hospital, Omuta (M.K., M.S.); Division of Internal Medicine, Toyama Hospital, Hitoyoshi (Y.T.); Division of Cardiology, Chikugo City Hospital, Chikugo (H.U.); and Division of Cardiology, Yame General Hospital, Yame (K.K.), Japan

Grants: This study was supported in part by a grant for the Science Frontier Research Promotion Centers from the Ministry of Education, Science, Sports and Culture of Japan.

This study was presented in the Late-Breaking Clinical Trials Session of the $76^{\text {th }}$ Annual Scientific Meeting of the Japanese Circulation Society, March 17, 2012, Fukuoka, Japan.

Mailing address: Hisashi Kai, MD, PhD, Department of Internal Medicine, Division of Cardio-Vascular Medicine, Kurume University School of Medicine, 67 Asahimachi, Kurume 830-0011, Japan. E-mail: naikai@med.kurume-u.ac.jp

ISSN-1346-9843 doi:10.1253/circj.CJ-12-1102

All rights are reserved to the Japanese Circulation Society. For permissions, please e-mail: cj@j-circ.or.jp 


\begin{tabular}{|c|c|}
\hline \multicolumn{2}{|l|}{ Characteristics } \\
\hline Combination regimen & $47(49.5)$ \\
\hline Age (years) & $69.5 \pm 10.2$ \\
\hline Men & $41(43.6)$ \\
\hline BMI & $24.1 \pm 3.5$ \\
\hline Morning SBP $(\mathrm{mmHg})$ & $149.9 \pm 7.9$ \\
\hline Evening SBP $(\mathrm{mmHg})$ & $138.2 \pm 10.9$ \\
\hline M-E difference $(\mathrm{mmHg})$ & $12.3 \pm 12.5$ \\
\hline Clinic SBP $(\mathrm{mmHg})$ & $146.6 \pm 13.2$ \\
\hline UACR (mg/g Cre $)^{\dagger}$ & $62.8(40.9-84.7)$ \\
\hline Uric acid (mg/dl) & $5.67 \pm 1.56$ \\
\hline eGFR $\left(\mathrm{ml} \cdot \mathrm{min}^{-1} \cdot 1.73 \mathrm{~m}^{-2}\right)$ & $69.9 \pm 15.0$ \\
\hline $\mathrm{K}(\mathrm{mmol} / \mathrm{L})$ & $4.34 \pm 0.40$ \\
\hline LDL-C (mg/dl) & $115.0 \pm 28.4$ \\
\hline HDL-C (mg/dl) & $59.0 \pm 14.8$ \\
\hline Triglycerides $(\mathrm{mg} / \mathrm{dl})^{\dagger}$ & $121.6(110.1-133.1)$ \\
\hline Hemoglobin $A_{1 c}(\%)$ & $5.57 \pm 0.60$ \\
\hline $\mathrm{BNP}(\mathrm{pg} / \mathrm{ml})^{\dagger}$ & $47.4(37.2-57.6)$ \\
\hline \multicolumn{2}{|l|}{ Comorbidity } \\
\hline Diabetes & $17(18.1)$ \\
\hline Dyslipidemia & $36(48.4)$ \\
\hline Coronary artery disease & $14(14.9)$ \\
\hline Cerebrovascular disease & $7(7.4)$ \\
\hline Smoking & $13(14.6)$ \\
\hline \multicolumn{2}{|l|}{ Co-prescribed drug } \\
\hline Ca channel blocker & $33(36.3)$ \\
\hline$a$-blocker & $5(5.3)$ \\
\hline$\beta$-blocker & $14(15.3)$ \\
\hline Loop diuretics & $1(1.1)$ \\
\hline
\end{tabular}

Data given as $n(\%)$, mean \pm SD or geometric mean $(95 \%$ confidence interval). ${ }^{+N a t u r a l}$ logarithm-transformed data were used for statistical analysis. BMI, body mass index; BNP, B-type natriuretic peptide; Cre, creatinine; eGFR, estimated glomerular filtration rate; HDL-C, high-density lipoprotein cholesterol; LDL-C, lowdensity lipoprotein cholesterol; M-E difference, morning-evening SBP difference; SBP, systolic blood pressure; UACR, urine albumin/ creatinine ratio.

receiving losartan/HCTZ combination than those receiving high-dose losartan. The original MAPPY study included some patients lacking one or more data point at the baseline and/or post-treatment assessment. The occurrence of missing data represents a potential source of bias in evaluating the relationship among UAE reduction, morning SBP reduction, and other factors. Accordingly, to investigate factors associated with UAE reduction, this subanalysis included only patients for whom complete data were available.

\section{Methods}

\section{Study Setting}

The MAPPY study (Clinical Trials Registration-Unique Identifier: NCT00795847) was a 3-month prospective, randomized, open-labeled, parallel-group, multicenter trial with blinded endpoint assessment. ${ }^{1}$ The institutional review boards or ethics committees of all participating institutions approved the study protocol. All patients gave written informed consent.

\section{Subjects}

The MAPPY study enrolled outpatients who had morning hypertension despite anti-hypertensive medications. ${ }^{1}$ Morning hypertension was defined as morning SBP $\geq 135 \mathrm{mmHg}$, diastolic blood pressure (DBP) $\geq 85 \mathrm{mmHg}$, or both ${ }^{2}$ on 7 different days during the 1-month run-in period. The exclusion criteria were as follows: secondary hypertension, malignant hypertension or uncontrolled hypertension (eg, DBP $\geq 120 \mathrm{mmHg}$ ), uncontrolled diabetes mellitus with $\mathrm{HbA}_{1 c} \geq 9.0 \%$, a history of gout or serum uric acid $\geq 8.0 \mathrm{mg} / \mathrm{dl}$, serum creatinine (Cre) $\geq 2.0 \mathrm{mg} / \mathrm{dl}$, serum potassium $(\mathrm{K}) \geq 5.5 \mathrm{mmol} / \mathrm{L}$, liver damage (eg, ALT or $\gamma$-GTP level $>3 \times$ the upper normal limit), use of a thiazide diuretic, use of an angiotensin II type I receptor blocker (ARB) or angiotensin-converting enzyme inhibitor (ACEI) exceeding the standard doses approved in Japan, and contraindications for thiazides or ARBs.

This subanalysis included only patients for whom complete data were available. Of 216 patients who were enrolled in the original MAPPY study, a total of 121 were excluded because the patients lacked data for one or more parameters, such as $\mathrm{UAE}$ and type B-natriuretic peptide (BNP), at the baseline and/or post-treatment assessment. Accordingly, 95 patients were enrolled in this study.

\section{Study Protocol}

The study protocol has been described elsewhere. ${ }^{1}$ Briefly, an anti-hypertensive drug was randomly switched to a $50-\mathrm{mg}$ losartan/12.5-mg HCTZ combination tablet (combination regimen) or 100-mg losartan (high-dose regimen) without washout period. If patients had been given an ARB or ACEI, the ARB or ACEI was switched to losartan/HCTZ combination or 100-mg losartan. If an ARB or ACEI had not been prescribed, one of the prescribed anti-hypertensives was changed to either of the test drugs. The prescription of anti-hypertensive agents was not changed during the 3-month treatment.

\section{Data Collection and Measurement}

The initial evaluation and laboratory tests were performed after written informed consent was obtained. Patients were instructed to perform home blood pressure (BP) self-measurement in the early morning (within $1 \mathrm{~h}$ after waking up, after urination, before dosing in the morning, before breakfast, after 1-2-min resting in a sitting position) and in the evening (before bedtime, after 1-2-min resting in a sitting position) every day, according to the Japanese Society of Hypertension Guidelines for the Management of Hypertension 2009 (JSH2009 guidelines). ${ }^{2}$ The first measurement taken each morning and evening were recorded. ${ }^{2}$ The measurements for the last 7 days preceding each clinic visit were averaged for the morning and evening BP. Cuff oscillometry was applied using electronic upper arm-cuff devices. The device type was unknown, but it was certificated that all devices conformed to the Association for the Advancement of Medical Instrumentation standards. Each patient used the same type of home BP monitoring device throughout the study. Clinic BP was recorded at each hospital visit using the sphygmomanometer after 5 min resting in a seated position at 1-2-min intervals. At least 2 measurements were averaged for the clinic BP measurement. ${ }^{2}$ The sum of morning SBP reduction and evening SBP reduction was defined as a parameter of the effects of all-day SBP reduction. Morning-evening SBP (M-E) difference was calculated by subtracting evening SBP from morning SBP.

Circulating biochemistry and UAE were measured in the morning in a fasting state at baseline and after 3 months of treatment. All assays were performed using commercially avail- 


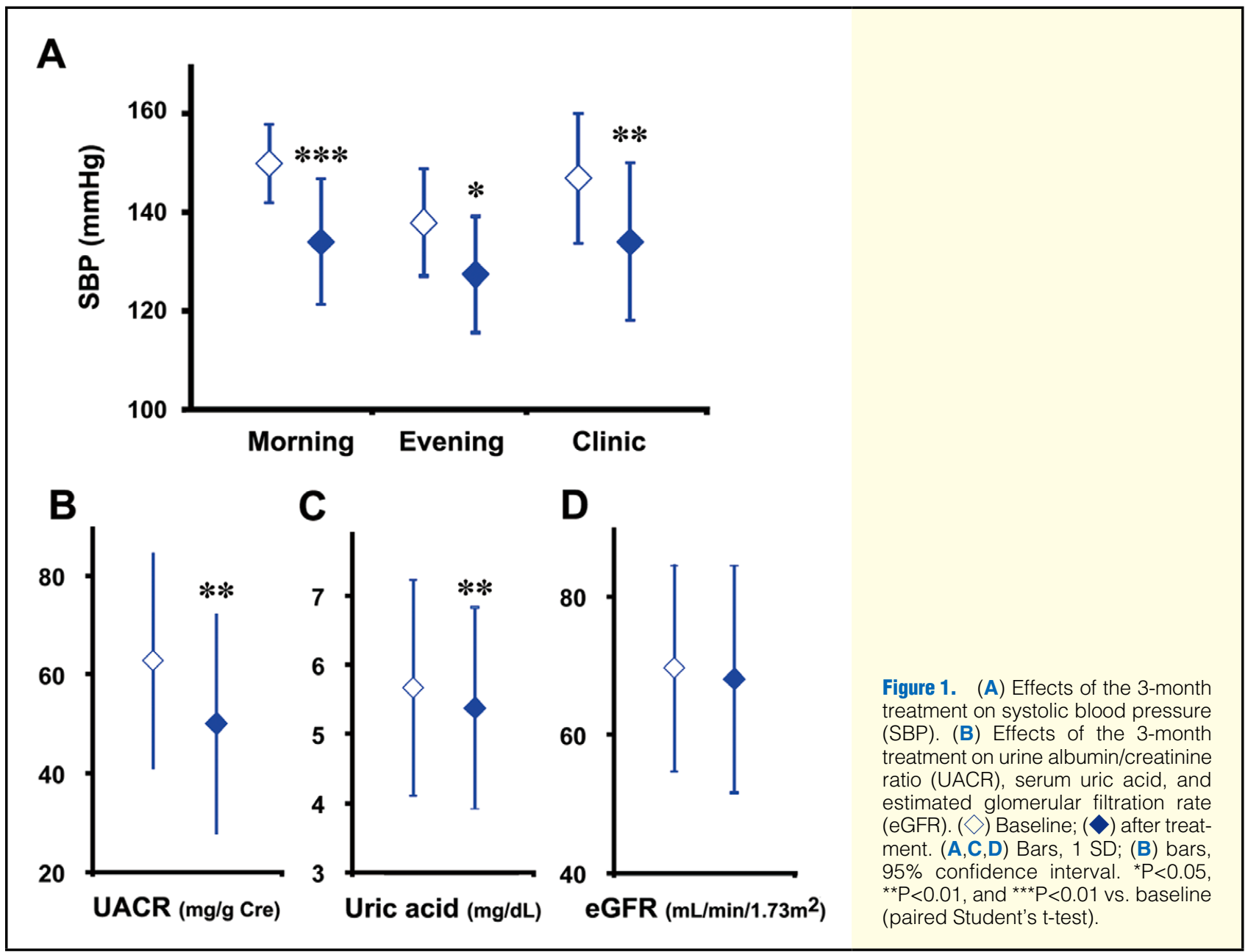

able laboratory. Estimated glomerular filtration rate (eGFR) was calculated using the Cockcroft-Gault equation modified for Japanese. ${ }^{2}$ UAE level was determined from a spot urine sample and expressed as urine albumin/Cre ratio (UACR; $\mathrm{mg} / \mathrm{g}$ Cre). ${ }^{3}$

\section{Statistical Analysis}

Statistical analysis was performed using IBM SPSS Statistics 18.0J (IBM Japan). Data are expressed as mean \pm SD. Because of skewed distributions, geometric mean (95\% confidence interval $[95 \% \mathrm{CI}]$ ) was used and natural logarithmic transformation was performed for baseline UACR, triglycerides, and BNP. Natural logarithm-transformed data were used for statistical analysis and then reconverted to anti-logarithm. Simple linear regression analysis was performed to investigate the relationships between \%UACR reduction and each of the following continuous variables: baseline levels and \%reductions of morning SBP, evening SBP, clinic SBP, morning SBP reduction+evening SBP reduction, M-E difference reduction; age, body mass index; baseline UACR; baseline level and $\%$ reduction of uric acid; and baseline level and \%change of $\mathrm{K}$, low-density lipoprotein cholesterol (LDL-C), high-density lipoprotein cholesterol (HDL-C), triglycerides, $\mathrm{HbA}_{1 \mathrm{c}}$, and BNP. Categorical variables were converted to dummy variables before the analysis. Two-sample t-test with equal variance or Welch's t-test with possible unequal variance was used to assess the association between \%UACR reduction and each of the categorical variables: treatment (combination regimen), male gender, diabetes, dyslipidemia, coronary artery disease, cerebrovascular disease, and ARB/ACEI use during the run-in period; Ca channel blocker use, $\beta$-blocker use, and $\alpha$-blocker use during the treatment period. Multiple linear regression analysis was performed using the significant variables. Differences for tertiles were evaluated using 1-way ANOVA for continuous variables and chi-square test for categorical variables. Analysis of covariance followed by post-hoc analysis was used to compare estimated \% UACR reduction according to tertiles of increasing \%morning SBP reduction. $\mathrm{P}<0.05$ was considered statistically significant.

\section{Results}

\section{Baseline Characteristics and Demographics}

This study included 95 patients (41 men; mean age, $69.5 \pm 10.2$ years) with morning hypertension based on home BP selfmeasurement (Table 1). At baseline, morning SBP, evening $\mathrm{SBP}$, and clinic SBP were $149.9 \pm 7.9 \mathrm{mmHg}, 138.2 \pm 10.9 \mathrm{mmHg}$, and $146.6 \pm 13.2 \mathrm{mmHg}$, respectively. Baseline UACR was $62.8 \mathrm{mg} / \mathrm{g}$ Cre (95\% CI: 40.9-84.7 mg/g Cre), and UACR was $<300 \mathrm{mg} / \mathrm{g}$ Cre (ie, microalbuminuria), in most subjects (94.8\%).

Anti-Hypertensive Treatment and Biochemical Parameters During the run-in period, an ARB or an ACEI was used in $93.7 \%$ of the study patients (data not shown). During the 


\begin{tabular}{|c|c|c|c|c|}
\hline & \multicolumn{2}{|c|}{ Simple } & \multicolumn{2}{|c|}{ Multiple } \\
\hline & $\boldsymbol{\beta}$ & P-value & $\beta$ & P-value \\
\hline Baseline UACR ${ }^{\dagger}$ & 0.300 & 0.003 & 0.341 & 0.001 \\
\hline \multicolumn{5}{|l|}{ Morning SBP } \\
\hline Baseline & -0.056 & 0.597 & - & - \\
\hline$\%$ Reduction & 0.335 & 0.002 & 0.353 & 0.001 \\
\hline \multicolumn{5}{|l|}{ Evening SBP } \\
\hline Baseline & -0.054 & 0.632 & - & - \\
\hline \% Reduction & 0.093 & 0.441 & - & - \\
\hline Morning SBP reduction + evening SBP reduction $\ddagger$ & 0.289 & 0.025 & - & - \\
\hline M-E difference reduction & 0.207 & 0.112 & - & - \\
\hline \multicolumn{5}{|l|}{ Clinic SBP } \\
\hline Baseline & -0.062 & 0.579 & - & - \\
\hline$\%$ Reduction & 0.073 & 0.482 & - & - \\
\hline$\%$ Uric acid reduction & -0.267 & 0.010 & -0.224 & 0.079 \\
\hline
\end{tabular}

${ }^{\dagger}$ Natural logarithm-transformed data were used for statistical analysis because of skewed distribution. $¥$ Omitted from multiple regression analysis because this variable was co-linear with \% morning SBP reduction. Abbreviations as in Table 1.

3 -month treatment, $49.5 \%$ of the patients were treated with the combination regimen (Table 1). Ca channel blocker, $\beta$-blocker, $\alpha$-blocker, and loop diuretics were co-prescribed in $37.9 \%$, $15.3 \%, 5.3 \%$, and $1.1 \%$, respectively, during the treatment period.

\section{Effects on Home and Clinic BP, UAE, and Biochemical Parameters}

The 3-month treatment with the combination regimen or highdose regimen significantly reduced morning SBP, evening SBP, and clinic SBP $(\mathrm{P}<0.001, \mathrm{P}<0.05$, and $\mathrm{P}<0.01$, respectively; Figure 1A). UACR and serum uric acid were significantly reduced after the 3-month treatment $(\mathrm{P}<0.01$ for both; Figures 1B,C). There were no differences in eGFR level (Figure 1D) and other parameters, such as M-E difference, $\mathrm{HbA}_{1 \mathrm{c}}$, lipids, and BNP, between the baseline and post-treatment assessments (data not shown).

\section{Significant Factors for UAE Reduction}

To determine the factors associated with UAE reduction, simple linear regression analysis was performed for continuous variables. A significant relationship was found between \%UACR reduction and \%morning SBP reduction, morning SBP reduction + evening SBP reduction, baseline UACR, or \% uric acid reduction (negatively), whereas \%UACR reduction did not have a significant correlation with \%evening SBP reduction, M-E difference reduction, \%clinic SBP reduction, and other variables (Table 2). In categorical variables, only the combination regimen produced a significant difference in UAE reduction $(\mathrm{P}=0.016)$.

Multiple linear regression analysis using \%morning SBP reduction, baseline UACR, and \%uric acid reduction as variables showed that baseline UACR and \%morning SBP reduction were independent determinants of \%UACR reduction $(\mathrm{P}=0.001$ for both; Table 2).

\section{Morning SBP Reduction and UAE Reduction}

To investigate correlation between morning SBP reduction and UAE reduction, we divided the patients into tertiles of increasing \%morning SBP reduction level (Table 3). There were significant differences in combination regimen, morning SBP reduction + evening SBP reduction, M-E difference reduction, \%UACR reduction, and \%uric acid reduction among the tertiles $(\mathrm{P}<0.001, \mathrm{P}<0.001, \mathrm{P}<0.001, \mathrm{P}=0.001$, and $\mathrm{P}=0.001$, respectively). After adjustments for $\mathrm{M}-\mathrm{E}$ difference reduction and \%uric acid reduction as covariates (model 1), estimated $\%$ UACR reduction was significantly correlated with \%morning SBP reduction (Figure $2 \mathrm{~A}$ ). Estimated \%UACR reduction was greater in the highest tertile than in the lowest tertile $(\mathrm{P}<0.05)$. Similar results were obtained even when baseline UACR was added into the covariates (model 2; Figure 2B).

\section{Effect of Treatment Regimen on UAE Reduction}

To investigate the effects of the difference in anti-hypertensive regimens on UAE reduction, subgroup analysis stratified by treatment regimen was performed. The combination regimen produced greater \%morning SBP reduction, greater morning SBP reduction + evening SBP reduction, and greater \%UACR reduction, as compared with the high-dose regimen $(\mathrm{P}<0.001$, $\mathrm{P}<0.01$, and $\mathrm{P}<0.05$, respectively). In contrast, the high-dose regimen produced greater \%uric acid reduction $(\mathrm{P}<0.001)$. Multiple linear regression analysis showed that baseline UACR and \%morning SBP reduction were independent determinants of \%UACR reduction in both subgroups (Table 4).

\section{Discussion}

Morning hypertension is an independent risk for cardiovascular events, such as stroke and myocardial infarction. ${ }^{4-8}$ On home BP self-measurement, morning hypertension was observed in $>60 \%$ of patients receiving anti-hypertensive drugs. ${ }^{9}$ It was suggested that the reduction in morning SBP reduced UAE and improved renal function in patients with diabetic and non-diabetic nephropathy. ${ }^{10,11}$ Standard treatment for morning hypertension, however, has not been established. An $\mathrm{ARB} / \mathrm{HCTZ}$ combination has complementary mechanisms of action leading to enhanced $\mathrm{BP}$ reduction, ${ }^{12}$ whereas high-dose ARB exhibits cardiovascular and renal protective effects. ${ }^{13}$ Both treatments are well tolerated in hypertensive patients. ${ }^{14}$ Thus, we conducted the MAPPY study to compare the effi- 


\begin{tabular}{|c|c|c|c|c|}
\hline & $\mathrm{T} 1(\leq 6.1 \%)$ & T2 (6.2-12.9\%) & T3 ( $\geq 13.0 \%)$ & P-value \\
\hline $\mathrm{n}$ & 32 & 31 & 32 & - \\
\hline Combination regimen & $6(18.8)$ & $20(64.5)$ & $21(65.6)$ & 0.000 \\
\hline Age (years) & $67.8 \pm 10.7$ & $71.2 \pm 11.5$ & $68.7 \pm 8.1$ & 0.461 \\
\hline Men & $13(40.6)$ & $18(58.1)$ & $13(40.6)$ & 0.230 \\
\hline BMI & $24.1 \pm 2.9$ & $23.6 \pm 3.4$ & $24.7 \pm 4.1$ & 0.577 \\
\hline \multicolumn{5}{|l|}{ Morning SBP } \\
\hline Baseline (mmHg) & $150.1 \pm 9.3$ & $149.2 \pm 6.8$ & $150.5 \pm 7.5$ & 0.821 \\
\hline \multicolumn{5}{|l|}{ Evening SBP } \\
\hline Baseline (mmHg) & $138.5 \pm 9.9$ & $136.9 \pm 11.7$ & $137.7 \pm 11.6$ & 0.884 \\
\hline \% Reduction & $4.4 \pm 8.3$ & $8.8 \pm 7.4$ & $10.0 \pm 9.9$ & 0.096 \\
\hline $\begin{array}{l}\text { Morning SBP reduction + evening SBP } \\
\text { reduction }(\mathrm{mmHg})\end{array}$ & $4.2 \pm 12.3$ & $26.4 \pm 9.0$ & $39.6 \pm 14.6$ & 0.000 \\
\hline \multicolumn{5}{|l|}{ M-E difference } \\
\hline Baseline (mmHg) & $12.9 \pm 12.5$ & $11.8 \pm 12.0$ & $12.1 \pm 13.4$ & 0.848 \\
\hline Reduction (mmHg) & $-8.3 \pm 13.7$ & $1.5 \pm 8.9$ & $11.2 \pm 14.3$ & 0.000 \\
\hline \multicolumn{5}{|l|}{ Clinic SBP } \\
\hline Baseline (mmHg) & $147.1 \pm 13.2$ & $147.8 \pm 12.9$ & $146.3 \pm 13.4$ & 0.915 \\
\hline \% Reduction & $7.2 \pm 11.0$ & $11.4 \pm 9.8$ & $6.1 \pm 10.4$ & 0.148 \\
\hline \multicolumn{5}{|l|}{ UACR } \\
\hline Baseline $(\mathrm{mg} / \mathrm{g} \mathrm{Cre})^{\dagger}$ & 75.5 (30.9-120.1) & $64.3(28.6-116.2)$ & $48.6(25.4-62.8)$ & 0.194 \\
\hline \% Reduction & $-30.9 \pm 70.2$ & $12.8 \pm 60.2$ & $28.1 \pm 48.8$ & 0.001 \\
\hline \multicolumn{5}{|l|}{ Uric acid } \\
\hline Baseline (mg/dl) & $5.64 \pm 1.53$ & $5.71 \pm 1.60$ & $5.18 \pm 1.02$ & 0.316 \\
\hline$\%$ Reduction & $15.2 \pm 17.7$ & $-3.5 \pm 20.9$ & $-2.1 \pm 22.8$ & 0.001 \\
\hline eGFR $\left(\mathrm{ml} \cdot \mathrm{min}^{-1} \cdot 1.73 \mathrm{~m}^{-2}\right)$ & $70.2 \pm 17.8$ & $68.7 \pm 15.9$ & $70.7 \pm 11.5$ & 0.879 \\
\hline $\mathrm{K}(\mathrm{mmol} / \mathrm{L})$ & $4.3 \pm 0.5$ & $4.4 \pm 0.5$ & $4.4 \pm 0.5$ & 0.496 \\
\hline LDL-C (mg/dl) & $116.6 \pm 33.6$ & $113.7 \pm 25.1$ & $116.5 \pm 27.9$ & 0.919 \\
\hline HDL-C (mg/dl) & $59.1 \pm 18.6$ & $60.4 \pm 12.0$ & $59.2 \pm 15.0$ & 0.947 \\
\hline Triglycerides $(\mathrm{mg} / \mathrm{dl})^{\dagger}$ & $146.3(120.9-171.7)$ & $103.1(84.7-121.6)$ & $120.9(103.5-138.3)$ & 0.140 \\
\hline Hemoglobin $A_{1 c}(\%)$ & $5.5 \pm 0.6$ & $5.5 \pm 0.6$ & $5.7 \pm 0.6$ & 0.227 \\
\hline $\mathrm{BNP}(\mathrm{pg} / \mathrm{ml})^{\dagger}$ & $45.4(29.1-61.8)$ & $53.2(31.1-75.3)$ & $45.3(28.3-62.3)$ & 0.784 \\
\hline \multicolumn{5}{|l|}{ Comorbidity } \\
\hline Diabetes & $4(12.5)$ & $6(19.4)$ & 7 (21.9) & 0.542 \\
\hline Dyslipidemia & $16(50.0)$ & $7(22.6)$ & $13(40.6)$ & 0.055 \\
\hline Coronary artery disease & $6(18.8)$ & $4(12.9)$ & $4(12.5)$ & 0.558 \\
\hline Cerebrovascular disease & $3(9.4)$ & $1(3.2)$ & $3(9.4)$ & 0.827 \\
\hline Smoking & $6(18.8)$ & $3(9.7)$ & $4(12.5)$ & 0.159 \\
\hline \multicolumn{5}{|l|}{ Co-prescribed drug } \\
\hline Ca channel blocker & $11(34.3)$ & $10(32.2)$ & $12(37.5)$ & 0.899 \\
\hline$a$-blocker & $3(9.4)$ & $2(6.5)$ & $5(15.6)$ & 0.229 \\
\hline$\beta$-blocker & $12(37.5)$ & $7(22.6)$ & $10(31.3)$ & 0.594 \\
\hline Loop diuretics & $0(0.0)$ & $1(0.3)$ & $0(0.0)$ & 0.363 \\
\hline
\end{tabular}

Data given as $\mathrm{n}(\%)$, mean \pm SD or geometric mean (95\% confidence interval). ${ }^{\dagger}$ Natural logarithm-transformed data were used for statistical analysis. Abbreviations as in Table 1.

cacy of these treatments in patients with morning hypertension. ${ }^{1}$ The MAPPY study has shown that a losartan/HCTZ combination induces a greater morning SBP reduction, the primary endpoint, than high-dose losartan $(-18 \mathrm{mmHg}$ vs. $-8 \mathrm{mmHg}, \mathrm{P}<0.001)$. With regard to the secondary endpoints, the combination regimen decreased UAE $(\mathrm{P}<0.05)$, whereas the high-dose regimen lowered serum uric acid $(\mathrm{P}<0.001)$. Either treatment did not change eGFR. Accordingly, we investigated the factors associated with the reduction in UAE, a surrogate marker of cardiovascular events, in patients with morning hypertension.

The most important finding of this study was that the reduc- tion in morning SBP and baseline UACR were independent determinants of UAE reduction in patients with morning hypertension (Table 2). This observation was consistent with a previous study showing that morning BP was a stronger predictor for UAE than clinic BP. ${ }^{15}$ It is interesting to note that morning SBP reduction was significantly correlated with UAE reduction even after adjustment for baseline UACR and other confounding factors (Figure 2). Moreover, the subgroup analysis showed that morning SBP reduction is an independent determinant of UAE reduction in both the combination regimen and high-dose regimen. Thus, it is suggested that morning SBP reduction is important for UAE reduction, irrespec- 
A

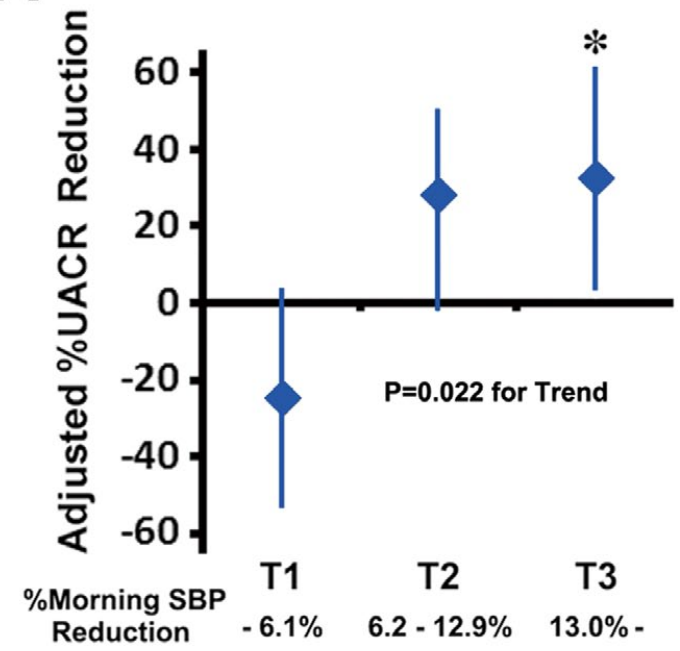

B

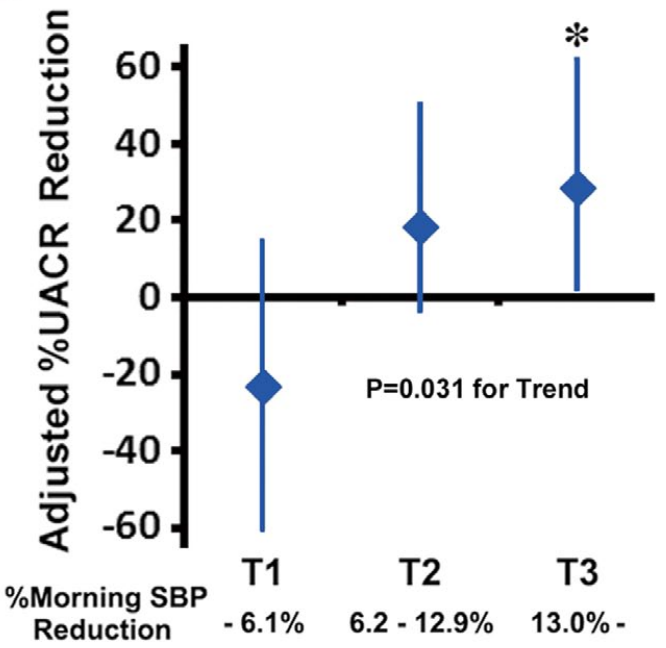

Figure 2. Estimated urine albumin/creatinine ratio (UACR) reduction vs. tertiles of increasing \%morning systolic blood pressure (SBP) reduction. (A) Model 1 was adjusted for morning-evening SBP (M-E) difference reduction and \%uric acid reduction as covariates. (B) Model 2 was adjusted for M-E difference reduction, \%uric acid reduction, and baseline UACR as covariates. Tertile (T) 1, \%morning SBP reduction $\leq 6.1 \%$; T2, 6.2-12.9\%; T3, $\geq 13.0 \%$. Bars, 95\% confidence interval. ${ }^{*}<<0.05$ vs. T1 (analysis of covariance followed by Bonferroni test). Because of skewed distributions, the natural logarithm-transformed baseline UACR data were used for statistical analysis and then reconverted to anti-logarithm.

\begin{tabular}{|c|c|c|c|c|c|c|c|c|}
\hline & \multicolumn{4}{|c|}{ Combination regimen $(n=47)$} & \multicolumn{4}{|c|}{ High-dose regimen $(n=48)$} \\
\hline & \multicolumn{2}{|c|}{ Simple regression } & \multicolumn{2}{|c|}{ Multiple regression } & \multicolumn{2}{|c|}{ Simple regression } & \multicolumn{2}{|c|}{ Multiple regression } \\
\hline & $\beta$ & P-value & $\beta$ & P-value & $\beta$ & P-value & $\beta$ & P-value \\
\hline Baseline UACR ${ }^{\dagger}$ & 0.400 & 0.005 & 0.383 & 0.006 & 0.321 & 0.026 & 0.346 & 0.022 \\
\hline \multicolumn{9}{|l|}{ Morning SBP } \\
\hline Baseline & -0.300 & 0.088 & & & 0.203 & 0.176 & & \\
\hline$\%$ Reduction & 0.433 & 0.005 & 0.440 & 0.002 & 0.298 & 0.040 & 0.297 & 0.048 \\
\hline \multicolumn{9}{|l|}{ Evening SBP } \\
\hline Baseline & -0.172 & 0.275 & & & 0.085 & 0.601 & & \\
\hline$\%$ Reduction & 0.138 & 0.436 & & & 0.241 & 0.150 & & \\
\hline $\begin{array}{l}\text { Morning SBP reduction + evening } \\
\text { SBP reduction }\end{array}$ & 0.152 & 0.440 & & & 0.275 & 0.127 & & \\
\hline \multicolumn{9}{|l|}{ M-E difference } \\
\hline Baseline & -0.031 & 0.851 & & & 0.018 & 0.917 & & \\
\hline Reduction & -0.355 & 0.064 & & & 0.031 & 0.865 & & \\
\hline \multicolumn{9}{|l|}{ Clinic SBP } \\
\hline Baseline & -0.102 & 0.496 & & & -0.228 & 0.119 & & \\
\hline \multirow[t]{2}{*}{$\%$ Reduction } & 0.073 & 0.625 & & & 0.080 & 0.587 & & \\
\hline & \multicolumn{4}{|c|}{$R^{2}=0.335$} & & & \multicolumn{2}{|c|}{$\mathrm{R}^{2}=0.187$} \\
\hline
\end{tabular}

†Natural logarithm-transformed data were used for statistical analysis because of skewed distribution. Abbreviations as in Table 1.

tive of treatment regimen.

We have to consider other factors. The use of diuretics might be a possible factor affecting UAE change, because diuretics deplete the circulating volume and decrease renal blood flow, which may reduce glomerular filtration. This, however, was not the case in the present study, because eGFR was not changed during the 3 -month treatment (Figure 1D). ${ }^{1}$ The present findings may be pertinent from the point of view that UAE not only reflects early renal damage, but is also a marker of endo- thelial dysfunction. ${ }^{16}$ Moreover, increased UAE level, even within the normal range, is a surrogate marker of cardiovascular events in both hypertensive patients and apparently healthy subjects. ${ }^{17,18}$

Morning SBP reduction + evening SBP reduction was significantly associated with \%morning SBP reduction on the basis of simple linear regression analysis (Table 2) and was significantly different among the tertiles of increasing \% morning SBP reduction (Table 3). Because morning SBP reduction + 
evening SBP was co-linear with \%morning SBP reduction, this variable was omitted from multiple regression analysis in Table 2 and from adjustment of \%UACR reduction in Figure 2. Also, there was a significant difference in \%uric acid reduction between the high-dose and combination regimens: the high-dose regimen, but not the combination regimen, decreased uric acid (\%uric acid reduction, $14.1 \pm 17.4 \%$ vs. $-7.5 \pm 18.8 \%$, respectively; $\mathrm{P}<0.001$ ), suggesting that \%uric acid reduction was associated with anti-hypertensive regimen. This might be related to the fact that losartan has a dose-dependent effect on the renal uric acid transporter- 1 inhibition to reduce uric acid level. ${ }^{19}$ Thus, we used \%uric acid reduction, but not combination regimen, as a significant variable for multiple linear regression analysis (Table 2) and a covariate for adjusting \%UACR reduction (Figure 2).

This study has several limitations. The number of patients studied was small. Because this study was a subanalysis of the MAPPY study, the balance of treatment regimen was not matched among the tertiles of \%morning SBP reduction. Next, care must be taken in interpreting the results of the subanalysis stratified by treatment regimen. The size of each subgroup was substantially small and the statistical power was not sufficient. Finally, the combination of ARB and Ca channel blocker is another alternative option for treatment of morning hypertension. ${ }^{20}$ Future studies should compare the effects on morning $\mathrm{SBP}$ and UAE between ARB/HCTZ combination and ARB/ Ca channel blocker combination.

\section{Conclusions}

Reduction in morning SBP was an independent determinant of $\mathrm{UAE}$ reduction in patients with morning hypertension. Thus, it is suggested that UAE reduction by morning BP reduction would be important for renal and cardiovascular protection in patients with morning hypertension.

\section{Acknowledgments}

This study was supported in part by a grant for the Science Frontier Research Promotion Centers from the Ministry of Education, Science, Sports and Culture of Japan. We thank Mari Shibata, Naoko Shiotani, Motoko Kakehashi, and Midori Ikeda for assistance with the manuscript.

\section{Disclosures}

None.

\section{References}

1. Ueda T, Kai H, Imaizumi T, on behalf of the MAPPY Study Investigators. Losartan/hydrochlorothiazide combination vs. high dose losartan in patients with morning hypertension: A prospective, randomized, open-labeled, parallel-group, multicenter trial. Hypertens Res 2012; 35: 708-714.

2. Ogihara T, Kikuchi K, Matsuoka H, Fujita T, Higaki J, Horiuchi M, et al. The Japanese Society of Hypertension guidelines for the management of hypertension (JSH 2009). Hypertens Res 2009; 32: 3 -
107.

3. Ewald B, Attia J. Which test to detect microalbuminuria in diabetic patients? A systematic review. Aust Fam Physician 2004; 33: $565-$ 567.

4. Muller JE, Tofler GH, Stone PH. Circadian variation and triggers of onset of acute cardiovascular disease. Circulation 1989; 79: 733-743.

5. Kario K, Ishikawa J, Pickering TG, Hoshide S, Eguchi K, Morinari M, et al. Morning hypertension: The strongest independent risk factor for stroke in elderly hypertensive patients. Hypertens Res 2006; 29: $581-587$.

6. Asayama K, Ohkubo T, Kikuya M, Obara T, Metoki H, Inoue R, et al. Prediction of stroke by home "morning" versus "evening" blood pressure values: The Ohasama study. Hypertension 2006; 48: 737-743.

7. Kario K, White WB. Early morning hypertension: What does it contribute to overall cardiovascular risk assessment? J Am Soc Hypertens 2008; 2: 397-402.

8. Kario K. Orthostatic hypertension: A measure of blood pressure variation for predicting cardiovascular risk. Circ J 2009; 73: 1002-1007.

9. Obara T, Ito K, Ohkubo T, Shibamiya T, Shinki T, Nakashita M, et al. Uncontrolled hypertension based on morning and evening home blood pressure measurements from the J-HOME study. Hypertens Res 2009; 32: $1072-1078$.

10. Suzuki H, Nakamoto H, Okada H, Sugahara S, Kanno Y. Self-measured systolic blood pressure in the morning is a strong indicator of decline of renal function in hypertensive patients with non-diabetic chronic renal insufficiency. Clin Exp Hypertens 2002; 24: 249-260.

11. Suzuki H, Kanno Y, Nakamoto H, Okada H, Sugahara S. Decline of renal function is associated with proteinuria and systolic blood pressure in the morning in diabetic nephropathy. Clin Exp Hypertens 2005; 27: 129-138.

12. MacKay JH, Arcuri KE, Goldberg AI, Snapinn SM, Sweet CS. Losartan and low-dose hydrochlorothiazide in patients with essential hypertension: A double-blind, placebo-controlled trial of concomitant administration compared with individual components. Arch Intern Med 1996; 156: 278-285.

13. Fogari R, Derosa G, Zoppi A, Preti P, Lazzari P, Destro M, et al. Effect of telmisartan-amlodipine combination at different doses on urinary albumin excretion in hypertensive diabetic patients with microalbuminuria. Am J Hypertens 2007; 20: 417-422.

14. Matsumura K, Arima H, Tominaga M, Ohtsubo T, Sasaguri T, Fujii K, et al. Does a combination pill of antihypertensive drugs improve medication adherence in Japanese? Circ J 2012; 76: 1415-1422.

15. Kamoi K, Ito T, Miyakoshi M, Minagawa S. Usefulness of home blood pressure measurement in the morning in patients with type 2 diabetes: Long-term results of a prospective longitudinal study. Clin Exp Hypertens 2010; 32: 184-192.

16. Stehouwer CD, Smulders YM. Microalbuminuria and risk for cardiovascular disease: Analysis of potential mechanisms. J Am Soc Nephrol 2006; 17: 2106-2111.

17. Hillege HL, Fidler V, Diercks GF, van Gilst WH, de Zeeuw D, van Veldhuisen DJ, et al. Urinary albumin excretion predicts cardiovascular and noncardiovascular mortality in general population. Circulation 2002; 106: 1777-1782.

18. Grimm RH Jr, Svendsen KH, Kasiske B, Keane WF, Wahi MM. Proteinuria is a risk factor for mortality over 10 years of follow-up: MRFIT Research Group: Multiple Risk Factor Intervention Trial. Kidney Int 1997; 63: S10-S14.

19. Enomoto A, Kimura H, Chairoungdua A, Shigeta Y, Jutabha P, Cha $\mathrm{SH}$, et al. Molecular identification of a renal urate anion exchanger that regulates blood urate levels. Nature 2002; 417: 447-452.

20. Miyauchi K, Yamazaki T, Watada H, Tanaka Y, Kawamori R, Imai Y, et al. Management of home blood pressure by amlodipine combined with angiotensin II receptor blocker in type 2 diabetes. Circ J 2012; 76: $2159-2166$. 